\title{
An Enhanced Positioning Scheme Based on Optimal Diversity for Mobile Nodes in Ubiquitous Networks ${ }^{\star}$
}

\author{
Seokyong Yang and Sekchin Chang \\ Dept. of Electrical and Computer Engineering, University of Seoul, Seoul, Korea \\ \{syuun, schang213\}@uos.ac.kr
}

\begin{abstract}
A lot of schemes have been proposed for the realization of u-city. Especially, most of the schemes are based on ubiquitous networks. For various u-city services to be available, mobile nodes can be employed in ubiquitous networks. However, an accurate positioning scheme is required for the practical usage of the mobile nodes in the ubiquitous networks. In this paper, an optimal diversity technique is proposed to improve the positioning performance. Simulation results indicate that using the optimal diversity scheme, the positioning performance can considerably be enhanced for mobile nodes in ubiquitous networks.
\end{abstract}

\section{Introduction}

A lot of attention has recently been paid to the establishment of the services such as intelligent disaster prevention, intelligent building management, intelligent health care, intelligent traffic control, and so on in metropolitan cities [1]. The services can be converged more efficiently and can be accessed more easily via ubiquitous networks [2]. The city which can offer the services based on ubiquitous networks is generally called u-city. Usually, the wireless sensor node is considered a realistic basis for the implementation of ubiquitous networks [3]. When the wireless sensor node is equipped with mobility, the node acts as a mobile node in ubiquitous networks. For the practical realization of the mobile node, the sensor node algorithm can be implemented in a cellular modem [4]. Using the implementation approach, the design limitation such as low cost and low power $[3,5]$ can be overcome since the node is able to utilize the resources of the cellular phone. Moreover, once the wireless sensor node is implemented in the cellular modem, the u-city services can easily be offered to the cellular phone holder in ubiquitous networks. However, an efficient positioning is an inevitable requirement for the practical use of the mobile node in ubiquitous networks. As proposed in [4], it is assumed in this paper that the positioning approach consists of location detection and location tracking. In this paper, an optimal diversity scheme is proposed to improve the positioning performance. The optimal scheme is based on multiple antennas. Especially, a novel structure and new

\footnotetext{
* This work was supported by Smart (Ubiquitous) City Consortium under Seoul R\&BD Program.
} 
message format are presented to efficiently implement the multiple antennas and to effectively acquire the optimal diversity, respectively. Simulation results indicate that the proposed diversity scheme can significantly improve the positioning performance for mobile nodes in ubiquitous networks.

\section{The Efficient Structure for Mobile Nodes with Multiple Antennas}

For the realistic implementation of the mobile node, the sensor node module can be included in a cellular modem [4]. In addition, the mobile node employs multiple antennas to achieve the optimal diversity. Fig. 1 illustrates the structure of the cellular modem to include the mobile node with multiple antennas [6]. Since most of the recent cellular modems are based on the system-on-chip

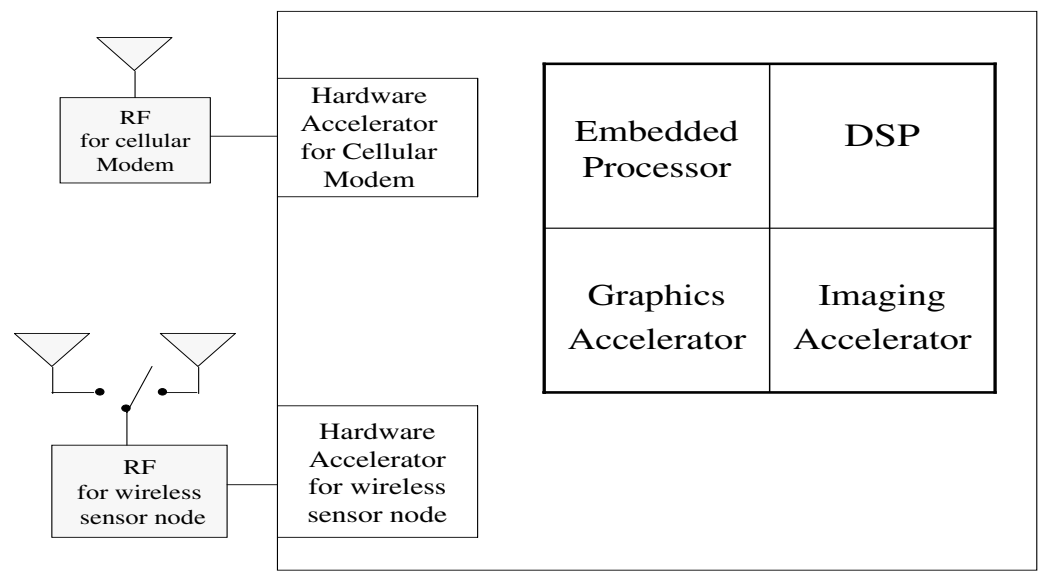

Fig. 1. The modem structure for the mobile node with diversity

(SoC) technology in the design, the cellular modem mainly consists of embedded processor, embedded DSP, hardware accelerators, graphics and imaging accelerators as shown in Fig. 1. In the view of such SoC structure, the hardware accelerator can be considered a kind of peripheral module. This indicates that the SoC structure relatively easily allows the addition of the hardware accelerators with low complexity. Therefore, the sensor node algorithm can be added in the cellular modem as a simple hardware accelerator since the algorithm exhibits relatively low complexity [7]. As illustrated in Fig. 1, the multiple antennas can be utilized to acquire the optimal diversity gain for the positioning of the mobile node. As indicated in [6], the modem structure utilizes only one RF module for two antennas in Fig. 1, which significantly alleviate the hardware overhead for the multiple antennas. Therefore, an efficient antenna switching and corresponding message format are also required for the optimal diversity combining. 


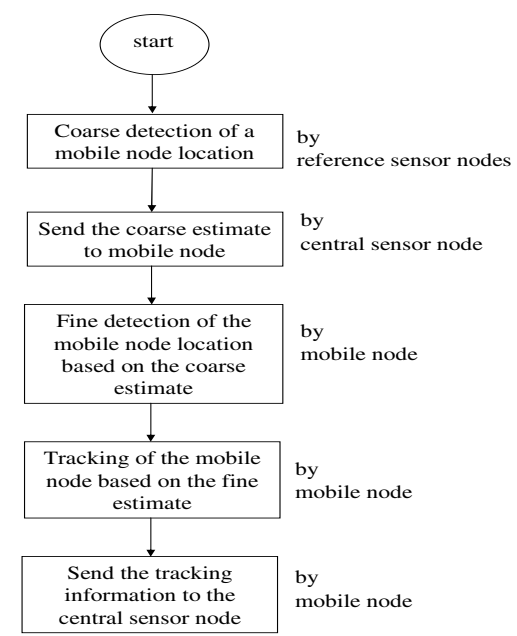

Fig. 2. The efficient positioning scheme for mobile nodes

\section{The Enhanced Positioning Scheme Based on Optimal Diversity for Mobile Nodes}

The mobile node can afford various u-city services to the phone user in ubiquitous networks. However, the location of the mobile node should accurately be detected and tracked to fully utilize the functionality of the node because the node can move in ubiquitous networks. Fig. 2 depicts the efficient positioning scheme for mobile nodes [4]. The positioning scheme consists of coarse and fine location detection, and location tracking in Fig. 2. As indicated in the figure, the reference sensor nodes roughly detect the location of the target mobile node. As the coarse detection, the trilateration scheme [8] is usually utilized by the reference sensor nodes which are selected from general wireless sensor nodes. Since the coarse detection usually exhibits simple computation, the general sensor nodes can perform the detection without high power consumption. However, the fine detection and the location tracking require highly intensive computation, which usually leads to high cost and high power consumption. Therefore, the target mobile node should perform the detection and the tracking by itself since the node can fully exploit the resources of the cellular phone. In the fine detection, the mobile node can increase the accuracy of the coarse estimate using an optimization technique such as the steepest descent algorithm [9]. Usually, the kind of algorithm exhibits fairly good performance when the initial value is close to the optimal value. If the coarse estimate can be used as the initial value, the algorithm can generate a fairly accurate estimate. For the use of the coarse estimate as the initial value in the fine estimation, the coarse estimate should be transmitted to the target mobile node by a central sensor node which is selected from the reference nodes. However, the received estimate usually includes the error due to the channel fading, which degrades the performance of 
the fine location detection. To overcome the performance degradation, the optimal diversity can be utilized in the mobile node. For the mobile node to achieve the optimal diversity gain on the modem structure of Fig. 1, the central node should transmit the coarse estimate using the proposed message format which is illustrated in Fig. 3. As shown in the figure, the message format includes

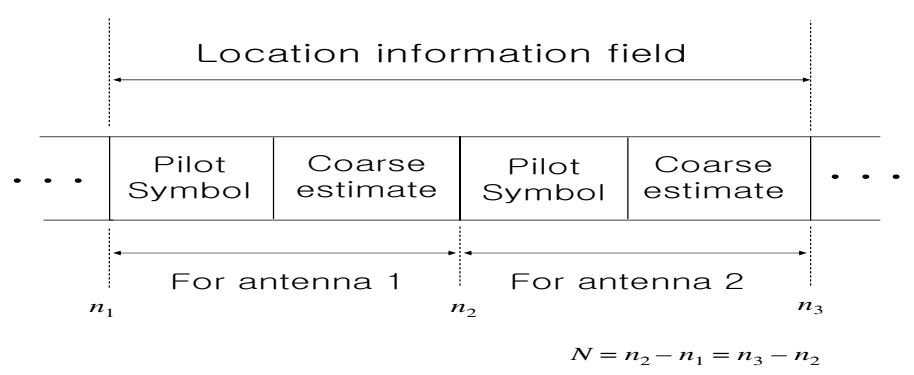

Fig. 3. The message format for the optimal diversity of mobile nodes

location information field which consists of the same two coarse estimation values and two pilot symbols. The first pilot symbol and the second pilot symbol are located at the discrete time-index of $n_{1}$ and $n_{2}$, respectively in the figure. As depicted in Fig. 3, the total time length for pilot symbol and coarse estimate is $N$. When the central sensor node transmits the coarse estimates in the message format, the received coarse estimate signal at the $k^{t h}$ antenna $r_{k}(n)$ is expressed as

$$
r_{k}(n)=\alpha_{k} \cdot s(n)+\eta_{k}(n)
$$

where $s(n)$ denotes the transmitted coarse estimation signal, and $\alpha_{k}$ and $\eta_{k}(n)$ indicate the channel parameter and additive white Gaussian noise (AWGN), respectively at the $k^{t h}$ antenna. In (1), the flat fading is considered the channel effect. In addition, in (1) $k=1$ when $n_{1} \leq n<n_{2}$, and $k=2$ when $n_{2} \leq$ $n<n_{3}$. In other words, the mobile node receives the first coarse estimate at the $1^{\text {st }}$ antenna, and then switches to the $2^{\text {nd }}$ antenna and receives the second coarse estimate at the $2^{\text {nd }}$ antenna. Then, the received coarse estimate signals are combined as follows:

$$
r(n)=w_{1} \cdot r_{1}(n)+w_{2} \cdot r_{2}(n)
$$

where $w_{k}$ denotes an optimal weighting coefficient for the $k^{\text {th }}$ antenna. For the optimal diversity gain in (2), the weighting coefficients, $w_{1}$ and $w_{2}$ are determined to maximize the signa-to-noise ratio (SNR) of $r(n)$. Using Cauchy's inequality, the SNR of $r(n)$ can be expressed as

$$
\frac{\sigma_{s}^{2}\left|\sum_{k=1}^{2} w_{k} \alpha_{k}\right|^{2}}{\sigma_{n}^{2} \sum_{k=1}^{2}\left|w_{k}\right|^{2}} \leq \frac{\sigma_{s}^{2} \sum_{k=1}^{2}\left|w_{k}\right|^{2} \sum_{k=1}^{2}\left|\alpha_{k}\right|^{2}}{\sigma_{n}^{2} \sum_{k=1}^{2}\left|w_{k}\right|^{2}}=\frac{\sigma_{s}^{2}}{\sigma_{n}^{2}} \sum_{k=1}^{2}\left|\alpha_{k}\right|^{2}
$$


where $\sigma_{s}^{2}$ and $\sigma_{n}^{2}$ denote signal power and noise power, respectively. Using the condition for the equality in (3), $w_{k}$ is determined as

$$
w_{k}=\alpha_{k}^{*}
$$

where $*$ denotes the complex conjugate. As indicated in (4), the channel parameters need to be estimated for the optimal coefficients. The channel estimation can be performed using the pilot symbols in the message format of Fig. 3. From (2) through (4), the weighing coefficients of (4) maximizes the SNR of the combined signal $r(n)$ of (2), which leads to the optimal diversity combining. Since the signal with the maximal SNR usually causes the best receiver performance, the mobile node can achieve the highest probability for decoding the received coarse estimate signal correctly using the proposed optimal diversity technique. This surely increases the estimation accuracy in the location detection because the detection searches the fine location position based on the decoded coarse estimate value. Therefore, it is concluded that the mobile node can enhance the estimation performance of the location detection using the optimal diversity.

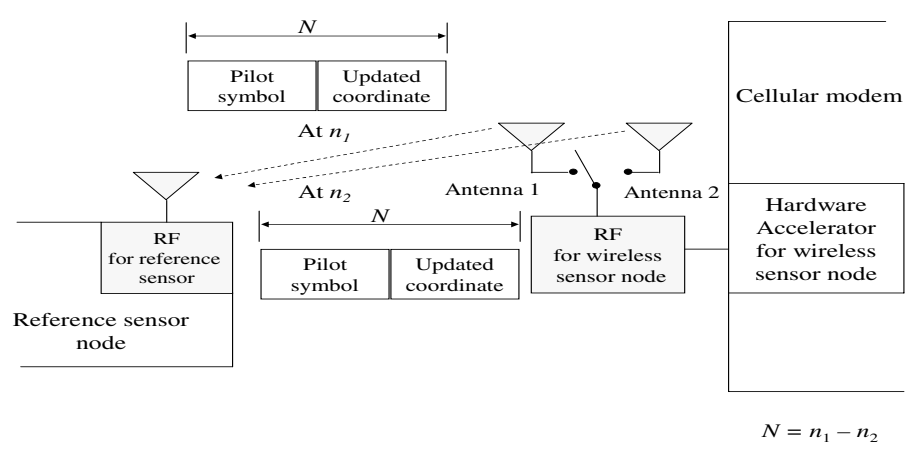

Fig. 4. The transmission of the updated estimate using optimal transmitter diversity in mobile nodes

If the mobile node is in motion, the fine estimate should continuously be updated in the location tracking as follows [4]:

$$
x^{(n+1)}=x^{(n)}+\hat{l} \cdot \triangle, y^{(n+1)}=y^{(n)}+\hat{m} \cdot \triangle
$$

where $x^{(n)}$ and $y^{(n)}$ are the updated coordinates of the mobile node at time index of $n$. In (5), $\hat{l}$ and $\hat{m}$ are the integer indices to indicate the motion direction and the motion amount of the mobile node. In addition, $\triangle$ denotes the coordinate incremental for tracking. Since high computation is usually required to determine $\hat{l}$ and $\hat{m}$, the mobile node also performs the location tracking by itself. Therefore, the mobile node should send the updated coordinate back to the central node whenever the update of (5) occurs. For the central node to decode the received 
coordinate more correctly, the optimal transmitter diversity can be utilized in the mobile node as shown in Fig. 4. In other words, the mobile node transmits the updated coordinate and the pilot symbol through the antenna 1 at the time index of $n_{1}$, and then switches to antenna 2 and transmits the same coordinate and the pilot symbol through antenna 2 at the time index of $n_{2}$. In Fig. 4, $N$ denotes the total time length of the updated coordinate data and the pilot symbol, and is defined as $n_{2}-n_{1}$. If the central node combines the received coordinate signals which are also weighted by the optimal coefficients of (4), the probability for correctly decoding the updated coordinate signal can be increased as in the case of the fine detection. Note that the pilot signals are also used for the channel estimation in the central node.

In the optimal diversity scheme, the additional overhead mainly includes simple addition and multiplication as shown in (2), which is minor in the system complexity. In addition, since the channel estimation is usually performed in most wireless systems [10], the additional complexity is not significant in the determination of the weighting coefficients of (4).

\section{Simulation Result}

Simulation results exhibit the effectiveness of the proposed optimal diversity scheme. For the simulation, the environment of Fig. 5 [4] is considered. This figure illustrates the moving of the mobile node from region 1 to region 2 . In addition, $2.4 \mathrm{GHz}$ flat fading and additive white Gaussian noise (AWGN) are assumed as the channel environments for the simulation.

For the performance evaluation of the location detection based on the optimal diversity scheme, the mean squared error (MSE) values between exact and estimated location position are given in Fig. 6. As shown in Fig. 6, the location detection with the optimal diversity achieves the gain of about $1 \mathrm{~dB}$ over the location detection without the diversity. For investigating the effects of the optimal transmitter diversity in the location tracking, Fig. 7 exhibits the updated coordinates that the central node decodes after receiving the coordinate signals

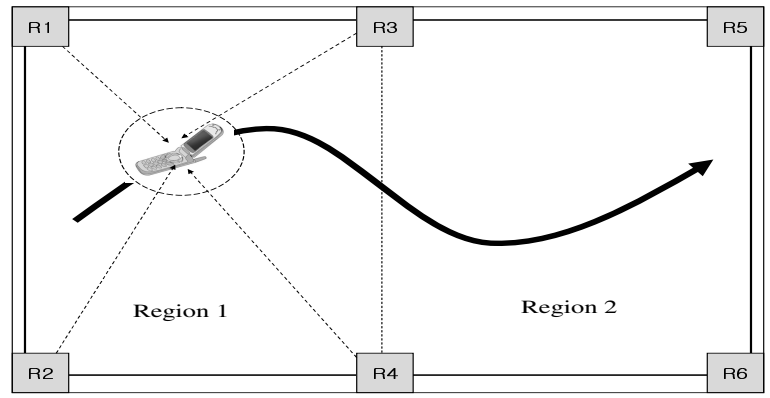

Fig. 5. The mobile node in ubiquitous networks 


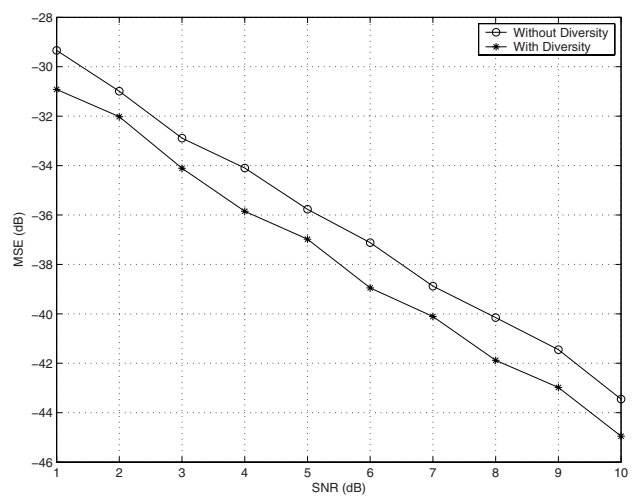

Fig. 6. The MSE performances for the location detection

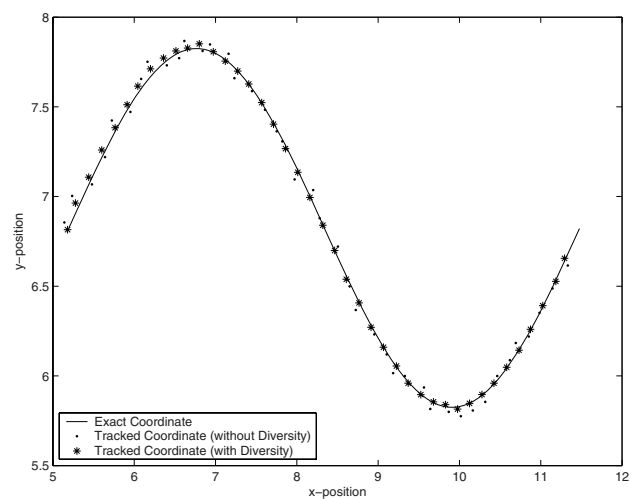

Fig. 7. The updated coordinates that the central node decodes

from the mobile node in motion under the environment of Fig. 5. As illustrated in Fig. 7, the decoded coordinates with the optimal diversity are much closer to the exact coordinates than those without the diversity.

\section{Conclusion}

In this paper, an optimal diversity scheme is proposed to improve the performances in locating and tracking the mobile node under ubiquitous networks. To acquire the diversity, the mobile node performs the antenna switching between two antennas, which considerably reduces the complexity overhead due to multiple antennas. For the performance enhancement in the location detection, the mobile node performs the optimal diversity combining based on the proposed message format. In addition, for more reliable transmission of the updated coordinates in the tracking, the mobile node utilizes the optimal transmitter diversity. 
The simulation results also reveal that the proposed diversity scheme can significantly enhance the detection and the tracking performances. For our further research, we will evaluate our proposed scheme in the real environments.

\section{References}

1. I. Akyildiz, W. Su, Y. Sankarasubramaniam, and E. Cayirci, Wireless Sensor networks: A Survey. Journal of Computer Networks 38 (2002) 393-422

2. N. S. Correal, and N. Patwari, Wireless Sensor networks: Challenges and Opportunities. Proc. of Virginia Tech Symp. Wireless Personal Comm. (2001) 1-9

3. E. H. Callaway Jr., Wireless Sensor networks: Architectures and Protocols. Auerbach. (2003)

4. P. Kim and S. Chang, An intelligent positioning scheme for mobile agents in ubiquitous networks for u-city. KES AMSTA 2007

5. C. M. Cordeiro and D. P. Agrawal, Ad Hoc \& Sensor Networks: Theory and Applications. World Scientific. (2006)

6. J. Lee and S. Chang, An Intelligent diversity scheme for accurate positioning of mobile agents for u-city. KES AMSTA 2007

7. IEEE Std 802.15.4: Wireless medium access control (MAC) and physical layer (PHY) specifications for Low-Rate Wireless Personal Area Networks (LR-WPANs). (2003)

8. N. Patwari, J. N. Ash, S. Kyperountas, A. O. Hero III, R. L. Moses, and N. S. Correal, Locating the nodes: cooperative localization in wireless sensor networks. IEEE Signal Processing Magazine (2005) 54-69

9. S. Haykin, Adaptive Filter Theory, 4th Edition. Prentice-Hall. (2001)

10. G. L. Stüber, Principles of Mobile Communication, Second Edition. Kluwer Academic Publishers. (2001) 